\title{
Análise dos preditores do estado psicológico das mães de crianças com Paralisia Cerebral
}

\section{Analysis of predictors of psychological status of mothers of children with cerebral palsy}

\author{
Patrícia Martins de Freitas* \\ Universidade Federal da Bahia - UFBA, Vitória da Conquista, Bahia, Brasil
}

\section{Carine Mendes Rocha**}

Universidade Federal do Recôncavo da Bahia - UFRB, Santo Antônio de Jesus, Bahia, Brasil

\section{Vitor Geraldi Haase***}

Universidade Federal de Minas Gerais - UFMG, Belo Horizonte, Minas Gerais, Brasil

\begin{abstract}
RESUMO
A sobrecarga física e emocional associada ao cuidado de crianças com patologias crônicas pode ocasionar prejuízos na vida cotidiana das mães e de outros membros da família. O sofrimento psicológico tem sido identificado e caracterizado, sendo ainda importante a investigação de variáveis preditoras desse fenômeno. O objetivo do estudo foi identificar preditores do sofrimento psicológico das mães de crianças com paralisia cerebral considerando: características da mãe (idade e escolaridade) percepção sobre o próprio estado emocional e saúde geral das mães, e o perfil comportamental. A análise baseou-se em análises descritivas, correlacionais e na análise de regressão múltipla. Participaram do estudo 122 mães de crianças com paralisia cerebral, em tratamento na Associação Mineira de Reabilitação, na cidade de Belo Horizonte. Os resultados demonstram que as variáveis preditoras do sofrimento psicológico foram: severidade dos comportamentos identificados como problemas; idade da criança e a escolaridade das mães. A saúde geral foi mais significativamente predita pelas seguintes variáveis: depressão, escolaridade e estresse relacionado ao cuidado da criança.
\end{abstract}

Palavras-chave: paralisia cerebral, sofrimento, comportamento.

\section{ABSTRACT}

The physical and emotional burden associated with caring for children with chronic diseases can cause damage in the daily lives of mothers and other family members. Psychological distress has been identified and characterized, and still important to investigate predictors of this phenomenon. The aim of these study was to identify predictors of psychological distress of mothers of children with cerebral palsy considering: characteristics of the mother (age and schooling) perception of own emotional state and general health of mothers, and the behavioral profile. 
The analysis was based on descriptive, correlational and multiple regression analyzes. The study included 122 mothers of children with cerebral palsy treated at Mineira Association of Rehabilitation in the city of Belo Horizonte. The results show that the predictors of psychological distress were: severity of behaviors identified as problems, age of the child and maternal schooling. The general health was most significantly predicted by the following variables: depression, education and child care-related stress.

Keywords: cerebral palsy, suffering, behavior.

\section{Introdução}

A investigação do estado emocional de mães de crianças com doenças crônicas ou progressivas tem sido o foco de diversos estudos, em função das possíveis interações entre o padrão psicológico das mães, comportamento das crianças e a qualidade de vida dos membros da família (Crnic, Fiedrich, \& Greenberg, 1983; Davis, Shelly, Waters, Boyd, Cook, \& Davern, 2010; Freitas, 2004; Hauser-Cram et al., 2001; Manuel, Naughton, Balkrisnan, Smith, \& Koman, 2003; Murphy, Christian, Caplin, \& Young, 2007; Sipal, Schuengel, Voorman, Van Eck, \& Becher, 2010; Taanila, Syrjälä, Kokkonen, \& Jarvelin, 2002; Thompson et al., 1994; Yim, Moon, Rah, \& Lee, 1996). A rotina de cuidados, associada aos tratamentos, pode provocar sobrecargas emocionais e a presença de sintomas como, por exemplo, ansiedade, depressão ou outros agravos para a saúde física (Byrne, Hurley, Daly, \& Cunningham, 2010; Davis et al., 2009; King, Teplicky, King, \& Rosenbaum, 2004; Parkes, Caravale, Marcelli, Franco, \& Colver, 2011). Entretanto, os preditores da sobrecarga emocional podem ser variáveis considerando as condições de cada doença. A sobrecarga emocional no cuidado de crianças com doenças crônicas pode ser resultado do perfil comportamental da criança, no estudo de Raina et al. (2005) foi verificado que comportamento hiperativo, distúrbios emocionais e de conduta explicam as alterações emocionais das mães. Entre diversas patologias crônicas, a Paralisia Cerebral (PC) possui características clínicas que impõem muitas mudanças para a família da criança, principalmente para as mães (Cheshire, Barlow \& Powell, 2010).

As alterações comportamentais especialmente o perfil externalizante de comportamento caracterizado por agitação, agressividade, quebra de regras e inadequações sociais é uma fonte de estresse no ambiente familiar (Miner \& Clarke-Stewart, 2008). A ocorrência conjunta de distúrbios de comportamento e condição clínica crônica em crianças pode ter um efeito negativo para o bem-estar das mães (Cheshire et al., 2010).

A PC é uma encefalopatia crônica não progressiva, mas geralmente mutável, de alterações motoras secundárias à lesão ou anomalias do cérebro em desenvolvimento (Camacho-Salas, Pallás-Alonso, De Las 
Heras \& Mateos-Beato, 2007; Hartley \& Thomas, 2003; Lai \& Yang, 2010; Lin, 2003; Monteiro, Jakabi, Palma, Pasin \& Meira, 2010; Mutch, Alberman, Hagberg, Kodama \& Perat, 1992). As características clínicas da PC exigem um tratamento sistemático de reabilitação, vinculando a criança e a mãe a uma rotina de atendimentos semanais com diferentes profissionais, vez que as habilidades motoras, cognitivas, e autocuidado, podem estar altamente comprometidas. Outra característica importante da PC é a frequência significativa de problemas de comportamento, destacamdo-se comportamentos impulsivos, petulância, desobediência, comportamento agressivo (Ribeiro, Porto, \& Vandenberghe, 2013; Sipal et al., 2010).

Por ser uma condição crônica, o diagnóstico da PC, pode ocasionar um impacto psicossocial para os pais da criança, vez que não apenas o indivíduo, mas, também, a família como um todo sofrerão alterações nas suas experiências diárias (Haase, Barreto, \& Freitas, 2009). As demandas familiares em geral podem ser maiores em relação aos cuidados destinados à criança clinicamente comprometida do que aos outros filhos sem transtornos do desenvolvimento, esse padrão foi verificado pelo estudo de (Hastings, 2002), investigando crianças autistas e suas famílias. Outros estudos evidenciam que as modificações na estrutura familiar atingem desde os recursos financeiros até as relações entre os membros (Crnic, Friedrich \& Greenberg, 1983; Glenn, Cunningham, Poole, Reeves \& Weindling, 2008; Hauser-Cram et al., 2001; Raina et al., 2005).

As modificações desencadeadas pelo processo adaptativo da família são acompanhadas de desajustes que podem estabelecer padrões emocionais disfuncionais (Freitas, Moreira, Rezende \& Haase, 2006; Lach et al, 2009). A criança com PC pode apresentar grande dependência por parte da mãe, sendo essa dependência física e/ou emocional. Essas especificidades do cuidado da criança com PC podem gerar sobrecarga das atividades ligadas ao cuidado e 0 tratamento da criança, o que pode implicar significativamente sobre a qualidade de vida das mães (de Oliveira \& Matsukura, 2013; Mobarak, Knan, Munir, Zaman, \& Mcconachie, 2000).

O impacto sobre a qualidade de vida de mães de crianças com PC tem sido demonstrado em diversos estudos (de Oliveira \& Matsukura, 2013; Raina et al., 2005). Existe um padrão semelhante sobre o estado psicológico de mães de crianças com transtornos do desenvolvimento. Entretanto, os determinantes desse padrão podem variar considerando as especificidades de cada condição clínica. Em (Freitas, 2004) foi demonstrado que mães de crianças com Distrofia Muscular tipo Duchenne (DMD) apresentavam maior sofrimento psicológico com o diagnóstico de seus filhos do que as mães de crianças como paralisia cerebral. Essa diferença foi explicada pelas especificidades das condições clínicas estudadas. No caso das mães 
de crianças com DMD a expectativa quanto ao futuro foi frequentemente apresentada como fator de sofrimento. Esses resultados sugerem a necessidade de outros estudos que investiguem os fatores que influenciam os padrões de sofrimento psicológico e prejuízos da qualidade de vida.

As condições clínicas envolvidas resultam em exigências para as famílias e podem funcionar como fatores de ameaça para o sistema familiar, provocando um ciclo de estresse e exigindo diversas adaptações (Freitas, Carvalho, Amarante, \& Haase, 2008). Este processo de adaptação psicológica dos familiares em relação à criança portadora de PC torna-se bastante complexo. A investigação sobre os fatores que contribuem para o sofrimento psicológico e prejuízos na qualidade de vida pode contribuir com 0 desenvolvimento de programas de intervenção que favorecem os processos adaptativos e a redução do estresse (Eisenhower, Baker, \& Blacher, 2005).

Um dos modelos teóricos que contribuem para a compreensão desse fenômeno é o modelo de estresse e coping de Lazarus e Folkman (1984). Segundo esse modelo o estresse assume uma perspectiva relacional e, portanto, o que é considerado estressor depende da avaliação subjetiva do indivíduo. A avaliação subjetiva é mediada pelos recursos pessoais e sociais. A partir dos resultados da avaliação primária, é iniciada a avaliação secundária que diz respeito a como lidar com o evento estressor, o que caracteriza a definição das estratégias de coping. As estratégias de coping são pensamentos e comportamentos que o indivíduo utiliza para lidar com as situações estressoras, e podem ser do tipo: centradas no problema e centradas nas emoções. Quando um indivíduo avalia uma determinada situação como inalterável utiliza mais o coping focado na emoção. Para as situações avaliadas como mais alterável do que inalterável, o indivíduo faz mais uso do coping focado no problema. As avaliações primárias e secundárias elaboradas pelo sujeito são diretamente influenciadas pelos fatores pessoais e sociais que irão interferir na definição do evento ao qual está submetido e nas estratégias de coping utilizadas.

Os recursos pessoais são descritos como características físicas ou psicológicas que vão favorecer de maneira positiva a adaptação frente ao evento, como a saúde física do sujeito, as crenças em que ele se apoia, além das habilidades que ele se utiliza para enfrentar a situação. Os recursos sociais são descritos como aqueles encontrados no ambiente contextual do sujeito, caracterizados por apoio e suporte social, ambiente familiar, situação econômica. A reavaliação constitui a terceira forma de avaliação realizada pelo sujeito. Nesta, o indivíduo modifica suas avaliações já realizadas conforme novas informações pessoais ou ambientais que foram obtidas, a partir daí 
reavalia-se as situações e elaboram-se novas estratégias de enfrentamento.

Esse modelo foi adaptado por Beresford (1994), especificando como evento potencialmente estressor ter uma criança com transtorno do desenvolvimento. Segundo Beresford (1994) o estresse poderia se minimizado ou maximizado de acordo com os recursos presentes. Segundo o autor, ter uma criança com transtornos do desenvolvimento pode ser um evento que ameaça, excede ou sobrecarrega seus recursos, prejudicando o bem estar, das mães, das crianças e da família. Essa perspectiva teórica contribuiu para os objetivos do estudo de investigar a relação entre o estado emocional das mães e os problemas de comportamento das crianças com PC, assim como a investigação das variáveis que podem interferir no estado emocional das mães.

Diversos estudos demonstram uma alta frequência de disfunções emocionais que podem ser classificadas dentro de um diagnóstico psicopatológico como ansiedade, depressão ou estresse em mães de crianças com transtornos do desenvolvimento (Davis et al., 2010; Pedersen, Parson \& Dewey, 2004; Phelps, Mccammon, Wuensch, \& Golden, 2009). A gravidade dos sintomas tende a diminuir com o tempo como demonstrado com adolescentes com PC (BrossardRacine et al., 2013.

Considerando a importância dos estudos sobre os aspectos psicossociais de ser mãe de uma criança com PC, o objetivo deste trabalho foi investigar preditores do estado emocional das mães dessas crianças, avaliando o nível de estresse, depressão, saúde geral, e avaliar a percepção das mesmas sobre o perfil comportamental das crianças. A análise da interação entre essas variáveis permite identificar fatores que interferem de forma mais significativa sobre a sobrecarga emocional das mães de crianças com PC.

\section{Método}

\subsection{Participantes}

Participaram do estudo 122 mães de crianças portadoras de PC, que recebiam tratamento na Associação Mineira de Reabilitação (AMR) em Belo Horizonte- MG. A idade média das mães foi de 33,77 anos ( $D P=9,09$ anos). A escolaridade foi medida em anos de escolarização formal, apresentando média de 8,14 anos $(D P=2,85)$. A idade média das crianças foi de 5,27 anos ( $D P=2,87$ anos).

\subsection{Critérios de I nclusão e Exclusão de participantes}


Os critérios de inclusão de participantes na amostra foram os seguintes: 1) Mãe de crianças com diagnóstico de PC; 2) A crianças deveria estar vinculada ao programa de reabilitação funcional da instituição (AMR); 3) Interesse e autorização manifestadas através do Termo Consentimento Livre e Esclarecido. Critérios de Exclusão: 1) Mães de crianças com outros Transtornos do Desenvolvimento que não fosse a PC; 2) Mães de crianças vinculadas à AMR, mas fora do programa de reabilitação funcional.

\subsection{Instrumentos}

Os instrumentos utilizados para a investigação do estado emocional das mães avaliaram o estresse relacionado ao cuidado das crianças com PC, a depressão, e a saúde geral. Para avaliação dos aspectos psicológicos, emocionais e saúde geral das mães utilizaram-se os instrumentos: Inventário de Depressão de Beck, Questionário de Estresse para Pais de Crianças com Transtornos do Desenvolvimento (QE-PTD) e Questionário de Saúde Geral. Outro foco foi a avaliação do perfil comportamental das crianças a partir da percepção da mãe. Para a avaliação da percepção das mães sobre o comportamento das crianças foram utilizados os seguintes instrumentos: Questionário de Comportamentos Importunos, Escala de Cotação de Hiperatividade e Questionário de Situações Domésticas (Barkley,1991). Esses três questionários fazem parte de um protocolo desenvolvido por Barkley, (1991, 1997) para avaliação do Transtorno de Déficit de Atenção por Hiperatividade (TDAH) e Transtorno do Comportamento Desafiador/Positivo (TDO). No presente estudo o protocolo não foi utilizado com a finalidade de encontrar um diagnóstico, mas verificar a presença de problemas de comportamentos que podem interferir na rotina diária das famílias de crianças com PC. Os instrumentos empregados foram adaptados para utilização no Brasil por Pinheiro, Haase, Del Prette, Amarante e Del Prette (2006).

Inventário de Depressão de Beck: O Inventário de Depressão de Beck (BDI) é um instrumento de autorrelato constituído por 21 itens sob a forma de afirmações. Cada um desses grupos possui quatro afirmações em que a pessoa deve responder qual a opção que esta de acordo com o seu estado na naquela determinada situação. Estas afirmações estão relacionadas com sinalizações dos sintomas referentes a estados depressivos daquela pessoa, variando em uma escala de 0 a 3 , na qual o respondente escolhe qual a afirmativa melhor corresponde a como se sente, considerando as duas últimas semanas incluindo o dia da aplicação. O escore é obtido a partir da soma dos itens e classificado dentro de uma escala crescente de gravidade da depressão mínimo, leve, moderado e grave. A classificação utilizada para os pontos de corte foi 0 a 11- mínimo; 12 a 19 (leve); 20 a 35 (moderado) e 36 a 63 
(grave). (Cunha, 2001). Os sintomas depressivos, possíveis de serem avaliados pelo $\mathrm{BDI}$, permitem que nas pesquisas de estresses sejam investigadas a presença de sintomas e atitudes associados à depressão e as relações desses com os níveis de estresse.

Questionário de Estresse para Pais de Crianças com Transtornos do Desenvolvimento - QE-PTD: O questionário foi adaptado da versão original do Questionnaire on Resources and Stress short-form (QRS-SF) (Friedrich, Greenberg \& Crnic, 1993). A versão brasileira é constituída de 32 itens e 4 fatores: Fator I - Restrições Comportamentais; Fator II - Sobrecarga emocional; Fator III - Pessimismo; Fator IV Características da Criança. O instrumento passou por estudo de adaptação, validação e confiabilidade apresentando resultados satisfatórios. O QE-PTD é um questionário objetivo do tipo Verdadeiro (V) ou Falso (F), no qual os pais devem pensar na criança com problemas de desenvolvimento enquanto preenchem o questionário. Por exemplo, “____ não se comunica com outras crianças de sua faixa etária." Em alguns itens existem lacunas em branco, ao ler esses itens os pais devem imaginar o nome da criança e responder $\mathrm{V}$ ou $\mathrm{F}$ (Freitas, 2004). No presente estudo a estrutura fatorial não foi considerada para as análises, pois foi considerado apenas o escore total que representa a medida do estresse.

Questionário de Saúde Geral (QSG): é um questionário de saúde física e psíquica, adaptado e validado para a população brasileira adulta (Pasquali, Gouveia, Andriola \& Ramos, 1996). O instrumento é composto por 60 perguntas, do tipo Likert de quatro pontos. Para a avaliação dos níveis de saúde ou das dimensões cognitivas e comportamentais avaliadas pelos valores, soma-se o valor do item respondido. Os itens apresentados pelo QSG visam avaliar as seguintes dimensões: sintomas depressivos; estresse físico e psicológico; características psicossomáticas; autoeficácia e distúrbios do sono. Estas dimensões são medidas por cinco fatores diferentes e um fator geral: fator I (estresse); fator II (desejo de morte); fator III (preocupação com o desempenho); fator IV (distúrbios do sono); fator $\mathrm{V}$ (fator somático) e fator $\mathrm{G}$ (fator geral).

Questionário de Comportamentos Importunos: O instrumento avalia a frequência de comportamentos nos quadros de Transtorno Desafiador Opositor e Transtorno de Déficit de Atenção e Hiperatividade (Barkley, 1991). Os comportamentos são descritos em 26 itens na forma de ações. As mães descrevem os comportamentos das crianças através das opções "Nenhum, ou Raramente", "De vez em quando", "Frequentemente" e "Muito frequentemente". 
Escala de Cotação de Hiperatividade: constitui-se por ser um instrumento objetivo formado por 14 itens relacionados aos aspectos comportamentais da hiperatividade. As respostas emitidas são direcionadas para as seguintes alternativas: "Quase nunca", "Um pouco", "Muito" e "Quase sempre” (Barkley, 1991).

Questionário de Situações Domésticas: o instrumento avalia a desobediência a ordens, normas elou regras em situações domésticas específicas. Considera dois aspectos relacionados ao comportamento da criança: o número de situações domésticas que os pais consideram como problemáticas e a gravidade de cada situação, dando uma pontuação de 0 a 9 . Os escores finais são expressos pelo número de situações domésticas consideradas problemáticas e pela média da gravidade, que representa a soma de pontos da gravidade dividida pelo número de situações (Barkley, 1991).

\section{Análise dos dados}

Os dados foram analisados em um pacote estatístico Statistical Packge of Social Science versão 11.5, seguindo os seguintes passos: 1) análise descritiva dos resultados sobre estado emocional das mães e sobre o comportamento das crianças com PC; 2) análise de correlação, utilizando o coeficiente de Pearson; 3) regressão múltipla, utilizando o método stepwise. O método utilizado foi o sptewise que considera a entrada de variáveis a partir da variância parcial apresentada; cada variável que entra no modelo é tratada como critério e as demais como covariáveis verificando o nível de significância. O procedimento é finalizado no momento em que não houver mais variáveis significativas para dar entrada no modelo (Mingotti, 2005). A escolha das variáveis para compor os modelos testados pela regressão múltipla foi feita a partir do poder de discriminação estabelecido a priori pela literatura. O nível de significância estabelecido foi de $p<0,05$.

\section{Procedimentos}

O estudo foi realizado na Associação Mineira de Reabilitação - AMR que é a terceira maior instituição de reabilitação na cidade de Belo Horizonte-MG. As mães de crianças com PC que estavam em atendimento no período foram convidadas através de uma cartaconvite. As que demonstraram interesse receberam o Termo de Consentimento Livre e Esclarecido de acordo com a aprovação do Comitê de Ética em Pesquisa (Parecer COEP no 227/02). Esse procedimento caracteriza a amostra como amostra de conveniência. 
Como critério de inclusão foi considerado a criança em reabilitação com diagnóstico de Paralisia Cerebral. Foram convidadas 300 mães, sendo que 57 não aceitaram e 21 desistiram sem finalizar a coleta. Essa amostra representou $74 \%$ das mães das crianças com PC atendidas na AMR. A coleta de dados era iniciada após leitura, esclarecimentos e assinatura do TCLE, e dividida em duas sessões de aproximadamente 60 minutos cada. A aplicação do protocolo foi realizada em uma sala disponibilizada pela AMR.

\title{
5 Resultados
}

Os resultados da análise descritiva identificam o perfil da amostra sobre as variáveis do estado emocional das mães e sobre o comportamento das crianças (ver Tabela 1).

\begin{abstract}
Tabela 1: Resultados descritivos do estado emocional das mães de crianças com PC e das escalas que avaliam a percepção das mães sobre o comportamento das crianças.
\end{abstract}

\begin{tabular}{|c|c|c|c|c|c|c|}
\hline $\begin{array}{c}\text { Foco da } \\
\text { investigação }\end{array}$ & Instrumentos & $\mathrm{n}$ & Mín & Máx & Média & $\begin{array}{l}\text { Desvio } \\
\text { Padrão }\end{array}$ \\
\hline \multirow{7}{*}{$\begin{array}{l}\text { Estado emocional } \\
\text { das Mães }\end{array}$} & Fator 1 QSG Stress & 122 & 1 & 3 & 2,07 & 0,59 \\
\hline & Fator 2 QSG Desejo de Morte & 122 & 1 & 3 & 1,28 & 0,47 \\
\hline & $\begin{array}{l}\text { Fator } 3 \text { QSG Preocupação com } \\
\text { o Desempenho }\end{array}$ & 122 & 1 & 3 & 2,00 & 0,49 \\
\hline & $\begin{array}{l}\text { Fator } 4 \text { QSG Perturbações do } \\
\text { Sono }\end{array}$ & 122 & 1 & 4 & 1,89 & 0,78 \\
\hline & Fator 5 QSG Somático & 122 & 1 & 3 & 1,90 & 0,53 \\
\hline & Fator G QSG Geral de Saúde & 122 & 1 & 3 & 1,90 & 0,45 \\
\hline & Inventário Beck de Depressão & 122 & 0 & 53 & 10,02 & 8,59 \\
\hline \multirow{6}{*}{$\begin{array}{l}\text { Problemas de } \\
\text { comportamento } \\
\text { das crianças }\end{array}$} & Número de Situações & 122 & 0 & 15 & 4,98 & 3,70 \\
\hline & Domésticas & & & & & \\
\hline & Severidade Média & 119 & 0 & 32 & 4,25 & 3,38 \\
\hline & $\begin{array}{l}\text { Questionário de } \\
\text { Comportamentos inoportunos }\end{array}$ & 106 & 0 & 66 & 17,64 & 14,26 \\
\hline & $\begin{array}{l}\text { Escala de Cotação da } \\
\text { Hiperatividade }\end{array}$ & 102 & 0 & 38 & 10,44 & 8,88 \\
\hline & QE-PTD* & 122 & 3 & 26 & 13,02 & 4,57 \\
\hline
\end{tabular}

Legenda: Fator 1 do QSG: Estresse; Fator 2 do QSG: Desejo de Morte; Fator 3 do QSG: Preocupação com o Desempenho; Fator 4 QSG: Pertubações do Sono; Fator 5 QSG:Somático; Fator G QSG: Fator Geral; IBD - Inventário Beck de Depressão; QE-PTD Questionário de Estresse para Pais de Crianças com Transtorno do Desenvolvimento; NSD- Número de Situações Domésticas; SM- Severidade Média; QCl- Questionário de Comportamentos Inoportunos; ECH- Escala de Cotação de Hiperatividade. 
Patrícia Martins de Freitas, Carine Mendes Rocha, Vitor Geraldi Haase Análise dos preditores do estado psicológico das mães de crianças com Paralisia

Os resultados apresentados pela amostra foram valores médios abaixo dos pontos de corte de cada instrumento, tanto para o estado emocional das mães quanto para os aspectos comportamentais das crianças. Esse resultado demonstra que, de modo geral, a amostra não apresenta características clínicas.

Tabela 2: Percentual de mães e crianças com algum tipo de alteração emocional.

\begin{tabular}{lc}
\hline \multicolumn{1}{c}{ Instrumentos } & $\%$ \\
\hline \multicolumn{1}{c}{ Avaliação do Estado Emocional das Mães } & \\
& \\
Fator 1 do QSG - Estresse & 9,80 \\
Fator 2 do QSG - Desejo de morte & 9,00 \\
Fator 3 do QSG - Preocupação com o Desempenho & 11,50 \\
Fator 4 do QSG - Perturbações do Sono & 17,20 \\
Fator 5do QSG - Queixas Somáticas & 13,10 \\
Fator 6 do QSG - Saúde Geral & 13,90 \\
Inventário Beck de Depressão & 16,40 \\
QE-PTD & 51,60 \\
& \\
Avaliação do Comportamento das Crianças com PC & \\
& \\
Número de Situações Domésticas & 6,24 \\
Severidade Média das Situações & 4,79 \\
Comportamentos Inoportunos & 16,32 \\
Escala de Cotação de Hiperatividade & 10,33 \\
\end{tabular}

Legenda: Fator 1 do QSG: Estresse; Fator 2 do QSG:Desejo de Morte; Fator 3 do QSG: Preocupação com o Desempenho; Fator 4 QSG: Pertubações do Sono; Fator 5 QSG:Somático; Fator G QSG: Fator Geral; IBD - Inventário Beck de Depressão; QE-PTD Questionário de Estresse para Pais de Crianças com Transtorno do Desenvolvimento; NSD- Número de Situações Domésticas; SM- Severidade Média; QCI- Questionário de Comportamentos Inoportunos; ECH- Escala de Cotação de Hiperatividade.

Na Tabela 2 são apresentados os resultados percentuais de mães acima do ponto de corte dos instrumentos utilizados. Demonstrou-se uma baixa frequência em relação aos aspectos gerais de saúde da mãe e sintomas depressivos. Entretanto, no que diz respeito aos valores investigados pelo questionário de estresse, apresentou-se uma alta frequência $(51,6 \%)$. A análise de correlação demonstrou 
que variáveis do estado emocional apresentam correlações entre si sendo essas as correlações moderadas e significativas que variam de 0,42 a 0,71 com $p<0,01$. O QE-PTD apresentou correlações moderadas com o Fator Estresse do QSG ( $r=0,48 ; p<0,001)$; com 0 Fator Geral de Saúde $(r=0,43 ; p<0,001)$ e com o IDB $(r=0,42$; $\mathrm{p}<0,001$ ).

Apesar de fracas outras correlações significativas foram identificadas e devem ser consideradas: correlação entre idade da mãe e comportamentos inoportunos $(r=0,23 ; p=0,02)$; Estresse Fator I do QSG e número de situações domésticas: $(r=0,28 ; p=0,01)$; escolaridade da mãe com os seguintes fatores do QSG, Saúde Geral $(r=0,29 ; p=0,01)$; Preocupação com o Desempenho $(r=0,25$ e $\mathrm{p}=0,002)$. A variável estresse avaliada pelo QE-PTD apresentou as seguintes correlações: com a idade da criança $(r=-0,38 ; p<0,001)$; Número de Situações Domésticas $(r=0,21 ; p<0,01)$ e Severidade Média $(r=0,27 ; p<0,001)$. Esses resultados foram uteis para a análise de regressão múltipla, auxiliando na composição dos modelos preditivos.

Os resultados da regressão múltipla demonstraram modelos preditivos para a saúde geral das mães e para o estresse relacionado à presença da criança com PC. Para analisar a saúde geral das mães foram incluídas as seguintes variáveis: idade da criança e da mãe, escolaridade da mãe, escores do Inventário Beck de depressão; escores do Questionário de Estresse para Pais de Crianças com Transtornos do Desenvolvimento; escores dos questionários que avaliam o comportamento da criança. Os resultados encontrados para a saúde geral as variáveis que compõe o modelo explicativo são os escores no Inventário Beck de Depressão; o escore do QE-PTD e a escolaridade da mãe. Para investigar o modelo que melhor prediz o estresse materno em relação ao transtorno do desenvolvimento, no caso a PC, foram incluídas as seguintes variáveis: idade da mãe e da criança; escolaridade da mãe; os escores do IDB; os escores do QSG e os escores de avaliação comportamento da criança. As variáveis com maior poder preditivo foram: severidade média dos comportamentos domésticos considerados inadequados; escolaridade das mães. Os resultados podem ser visualizados na Tabela 3. 
Patrícia Martins de Freitas, Carine Mendes Rocha, Vitor Geraldi Haase Análise dos preditores do estado psicológico das mães de crianças com Paralisia

Tabela 3: Regressão Múltipla para o estresse específico associado PC (QEPTD) e para a saúde geral (Fator G do QSG) das Mães.

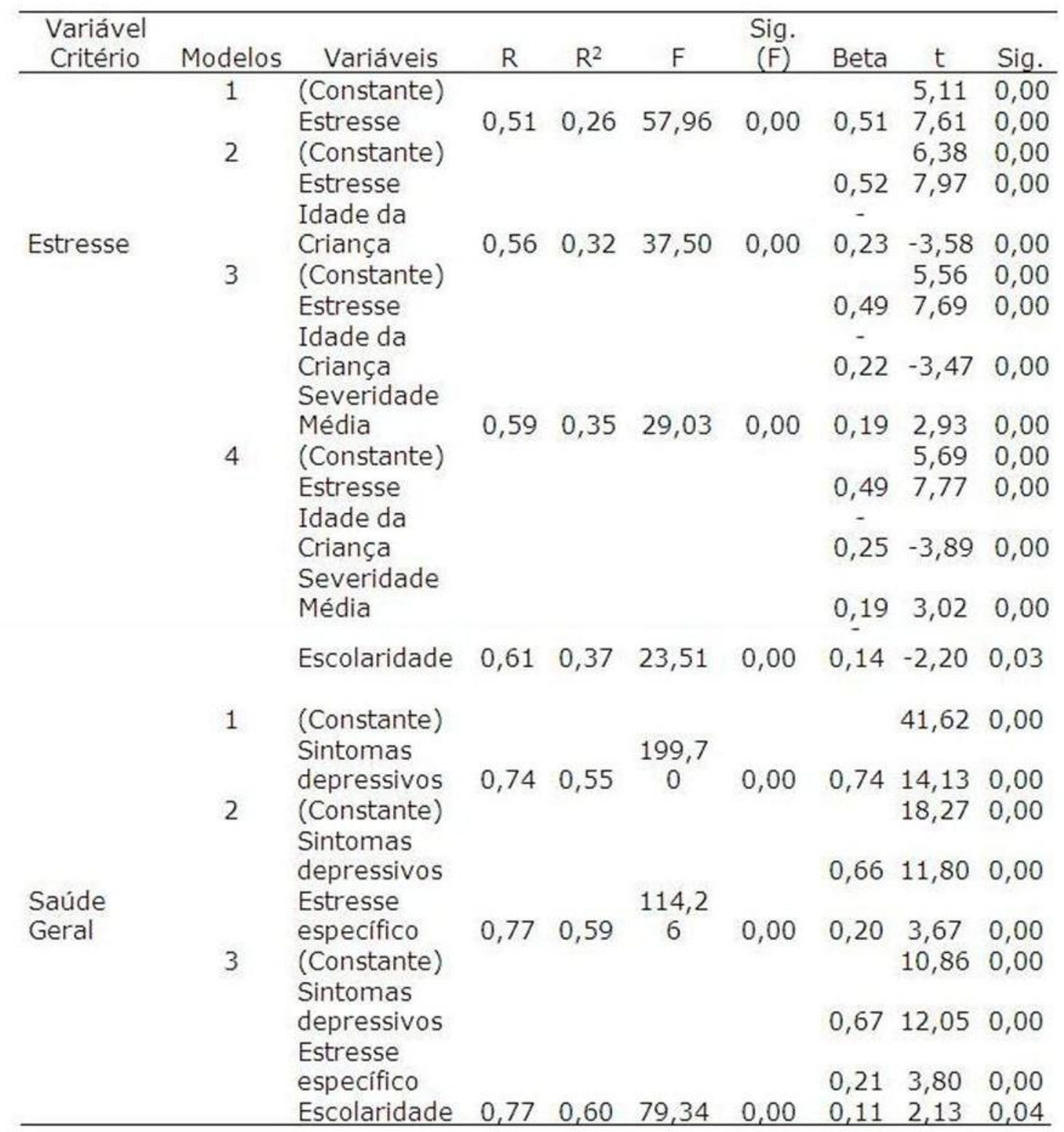

\section{Discussão}

As características emocionais de mães de crianças com PC têm sido demonstradas em diversos estudos que evidenciaram a maior vulnerabilidade para depressão e ansiedade como efeitos do estresse (Davis et al., 2009; Parkes et al., 2011). O estresse materno na PC tem sido explicado por diferentes variáveis que incluem aspectos sociodemográficos, assim como, características da mãe, perfil comportamental da criança (Mobarak et al., 2000) tempo de cuidado e recursos de enfrentamento (coping). A investigação a partir de 
modelos de predição tem sido um direcionamento para melhor explicar o estado emocional das mães (Parkes et al., 2011).

No presente estudo, o objetivo foi a investigação das variáveis preditoras do sofrimento psicológico, muitas vezes, presente em mães de crianças com PC, investigando a contribuição do perfil comportamental das crianças para o modelo explicativo do estresse. Os resultados encontrados demonstram que existe uma relação entre o estado emocional das mães, sua saúde geral e o comportamento das crianças com PC. Ketelaar, Volman, Gorter e Vermeer (2008) encontraram resultados que evidenciam a influência dos comportamentos mal adaptados das crianças com PC sobre 0 estresse materno. Resultado semelhante já tinha sido evidenciado no estudo de Mobarak et al., (2000). Outro preditor importante tem sido as características clínicas da PC, como, por exemplo, deficiência intelectual, comunicacional e dores que explicaram $12 \%$ da variância apresentada nas medidas de estresse dos pais investigadas por Parkes et al. (2011). Os autores encontraram altos níveis de estresse em pais de crianças com PC, cinco vezes mais do que na população geral, evidenciando a relevância clínica da sobrecarga emocional dos familiares de crianças com PC.

A presença na família de uma criança com transtorno do desenvolvimento é um estressor em potencial para as mães que, geralmente, assumem a responsabilidade do cuidado, exigindo adaptações constantes (Gallimore, Weisner, Kaufman \& Bernheimer, 1989; Schuengel, et al., 2009). As mães precisam reorganizar as suas tarefas diárias, de modo que venham potencializar os ganhos e minimizar as perdas (Freitas, et al., 2006). As mães e familiares de crianças portadoras de PC vivenciam algumas alterações em seu contexto, em função da rotina intensa de cuidados que pode representar uma sobrecarga (Davis et al., 2010). Esse padrão pode ocasionar problemas emocionais, levando a um comprometimento psicológico das mães que podem estar associados aos problemas de comportamento das crianças com PC (Skok, Harvey \& Reddihough, 2006).

As análises de correlações foram sugestivas de possíveis interações entre as variáveis, considerando inclusive a importância de variáveis sociodemográficas como, a idade da criança, idade das mães e escolaridade das mães. A escolaridade das mães também demonstrou correlação positiva com a saúde geral, preocupações com o desempenho e perturbações do sono. Uma possível interpretação desse resultado é que as mães com maior escolaridade podem ter expectativas mais altas em relação ao seu desempenho, o que pode afetar o sono e saúde geral. Segundo Hastings (2002), a expectativa das mães sobre o seu desempenho como cuidadora se mostrou uma variável importante para a investigação de sobrecarga emocional. 
O estresse específico associado a presença da criança com PC demonstrou relação inversamente proporcional com a idade da criança, quanto maior o estresse das mães, menor a idade das crianças e vice-versa. Esse resultado pode ser explicado por duas hipóteses: a menor idade da criança apresentar uma proximidade com o diagnóstico que é uma fase de alto nível de estresse (Taanila, et al., 2002); e a menor idade da criança localizar temporalmente uma fase de baixa adaptação ou acomodação (Beresford, 1994; Gallimore et al., 1989; Pitman \& Matthey, 2004; Sigurdardottir et al., 2010). Em relação à idade, o mesmo resultado foi encontrado no estudo de Brossard-Racine et al. (2013) que mostrou que adolescentes com PC têm menos problemas de comportamento e distúrbios emocionais do que crianças com PC. O avanço da idade pode ocasionar melhora no comportamento das crianças com PC, assim como a família pode apresentar melhores condições para lidar com os desafios, diminuindo o estresse e a percepção negativa dos pais sobre a criança. Esse resultado também foi encontrado no estudo de Glenn et al. (2008) que evidenciou que os níveis elevados de problemas de comportamento reduzem com o aumento da idade.

O estresse materno e o comportamento das crianças com PC são variáveis que se correlacionam. No presente estudo foi encontrada correlação positiva entre o estresse e o número de situações domésticas consideradas problemáticas, assim como, a gravidade das mesmas. Essa relação tem sido demonstrada em diversos estudos que investigam o estresse materno e o comportamento das crianças com algum transtorno do desenvolvimento. Entretanto, ainda existem limitações sobre os determinantes dessa relação (Ketelaar et al., 2008; Miodrag \& Hodapp, 2010; Nereo, Fee, \& Hinton, 2003).

A análise de regressão múltipla demonstrou resultados que contribuem para a investigação de quais são as variáveis que podem predizer o estresse de mãe de crianças com PC, assim como quais as variáveis preditivas do estado de saúde geral. Através desse estudo, demonstrou-se que o modelo que melhor explicou estresse associado à presença da criança com PC é composto pelas seguintes variáveis: sobrecarga emocional e escolaridade da mãe, a gravidade dos problemas de comportamento no ambiente doméstico e a idade da criança. Esse modelo confirma outros resultados da literatura sobre o processo de adaptação das mães, sendo os maiores níveis de estresse explicados não pelo transtorno do desenvolvimento em si, mas sim pelo impacto que os mesmos podem ter sobre 0 comportamento social da criança (Brossard-Racine et al., 2012; Manuel et al., 2003; Nereo et al., 2003; Parkes et al., 2008). Os problemas de comportamento das crianças com PC e outros transtornos do desenvolvimento explicam parte do estresse percebido pelas mães. Esse resultado está de acordo com o estudo de Sofronoff e Farbotko, (2002) que demonstrou que a redução do estresse 
materno contribui para a percepção de autoeficácia, aumentando a capacidade dos pais para manejar comportamentos inadequados. Outro resultado importante da análise de regressão múltipla foi identificação de variáveis que podem predizer o bem-estar físico e psicológico que nesse estudo foram mensurados pelo escore geral do QSG. A saúde mental de mães de crianças com PC apresenta vulnerabilidade para sintomas depressivos e de ansiedade (Parkes et al., 2011) Sobre os indicativos de prejuízos da saúde geral das mães foi possível demonstrar que as variáveis: escolaridade, os sintomas depressivos e estresse específico ocasionado pelos cuidados com a criança com PC são preditoras explicativas. A escolaridade pode estar associada aos níveis de exigência e expectativas quanto ao desempenho que as mães com maior escolaridade podem apresentar. As demais variáveis preditoras do modelo são convergentes com outros estudos que demonstram que o estresse e sintomas depressivos resultam em problemas de saúde para as mães (Lach et al., 2009, Mobarak et al., 2000; Hauser-Cram et al., 2001).

$O$ presente estudo também contribuiu para evidenciar a aplicabilidade do QE-PTD, um instrumento novo, porém consistente para a avaliação do estado emocional de pais de crianças com transtornos do desenvolvimento, por avaliar o estresse associado às especificidades de lidar com crianças clinicamente comprometidas. Os resultados encontrados foram semelhantes ao seu estudo inicial sobre sua validade convergente com o Inventario Beck de Depressão e Questionário de Saúde Geral de Goldeberg (Freitas, 2004).

Os resultados do presente estudo contribuem para o melhor direcionamento de programas de intervenção que tenham como foco - bem-estar das mães de crianças com PC ou da criança, demonstrando a necessidade de abordar os dois eixos, ou seja, o comportamento da criança e o estado emocional das mães (Freitas, Carvalho, Leite, \& Haase 2005; King et al., 2004). Apesar de contribuir com a investigação do estresse materno associado aos cuidados com a criança com PC consideramos a necessidade de novos estudos que utilizem outras variáveis como a participação ou não do cônjuge nos cuidados da criança, o número de filhos na família e a relação entre os irmãos e a contribuição da escolarização da criança. A adaptação e o bem-estar de mães de crianças com PC podem ter diversos efeitos benéficos, como mães mais produtivas, com menos problemas de saúde física e mental; famílias menos desajustadas; e principalmente o efeito no desenvolvimento da criança, incluindo o processo de reabilitação.

\section{Referências}


Barkley, R. A. (1991). Attention-defict hyperactivity desorder. A clinical workbook. New York: Guilford Press.

Barkley, R. A. (1997). Defiant children: Parent-teacher assignments. New York: Guilford Press.

Beresford, B. A. (1994). Resources and strategies: how parents cope with the care of a disabled child. Journal of Child Psychology and Psychiatry, 35(1), 171-209.

Brossard-Racine, M., Hall, N., Majnemer, A., Shevell, M. I., Law, M., Poulin, C., \& Rosenbaum, P. (2012). Behavioural problems in school age children with cerebral palsy. European Journal of Paediatric Neurology, 16(1), 35-41.

Brossard-Racine, M., Waknin, J., Shikako-Thomas, K., Shevell, M., Poulin, C., Lach, L., \& Majnemer, A. (2013). Behavioral difficulties in adolescents with cerebral palsy. Journal of child neurology, 28(1), 27-33.

Byrne, M. B., Hurley, D. A., Daly, L., \& Cunningham, C. G. (2010). Health status of caregivers of children with cerebral palsy. Child: Care, Health and Development, 36(5), 696-702.

Camacho-Salas, A., Pallás-Alonso, C. R., De La Cruz-Bértolo, J., De Las Heras, R. S., \& Mateos-Beato, F. (2007). Paralises cerebral: concepto y registros de base poblacional. Revista de Neurología, 45(8), 503-508.

Cheshire, A., Barlow, J. H., \& Powell, L. A. (2010). The psychosocial well-being of parents of children with cerebral palsy: a comparison study. Disability and Rehabilitation, 32(20), 16731677.

Cunha, J. A.(2001). Manual da Versão em Português das Escalas Beck. São Paulo: Casa do Psicólogo.

Crnic K. A., Friedrich W. N., \& Greenberg M. T. (1983) Adaptation of families with mentally retarded children: a model of stress, coping, and family ecology. American Journal of Mental Deficiency 88(2), 125-138.

Davis, E., Shelly, A., Waters, E., Mackinnon, A., Reddihough, D., Boyd, R., \& Graham, H. K. (2009). Quality of life of adolescents with cerebral palsy: perspectives of adolescents and parents. Developmental medicine \& child neurology, 51(3), 193-199.

Davis, E., Shelly, A., Waters, E., Boyd, R., Cook, K., \& Davern, M. (2010). The impact of caring for a child with cerebral palsy: Quality of life for mothers and fathers. Child: Care, Health and Development, 36(1), 63-73.

De Oliveira, A. K. C., \& Matsukura, T. S. (2013). Estresse e apoio social em cuidadores de crianças com paralisia cerebral/Stress and social support in caregivers of children with cerebral palsy. Cadernos de Terapia Ocupacional da UFSCar, 21(3), 493-513.

Eisenhower, A. S., Baker B. L., \& Blacher, J. (2005). Preschool children with intellectual disability: syndrome specificity, 
behaviour problems, and maternal well-being. Journal of Intellectual Disability Research, 49(9), 657-671.

Freitas, P. M. (2004). Adaptação Psicossocial de Mães de Crianças com Transtornos do Desenvolvimento: Uma Comparação Entre a Distrofia Muscular Progressiva Tipo Duchenne e a Paralisia Cerebral. Dissertação de mestrado. Universidade Federal de Minas Gerais, Belo Horizonte, Brasil.

Freitas, P. M., Carvalho, R. C. L., Leite, M. R. S. D. T., \& Haase, V. G.(2005). Modelo exploratório da relação entre o estresse materno e a inclusão escolar de crianças com paralisia cerebral. Arquivos Brasileiros de Psicologia, 57(1), 46-57.

Freitas, P. M., Moreira, P. P. C. M. S., Rezende, L. L., \& Haase, V. G. (2006). Qualidade de vida de mães de crianças com paralisia cerebral. Arquivos Brasileiros de Paralisia Cerebral, 2(5), 35-39.

Freitas, P. M., Carvalho, R. C. L., Amarante, C. L. D., \& Haase, V. G. (2008). Efeito de um Programa de Intervenção para Mães de Crianças com Paralisia Cerebral: Um Estudo Exploratório. Interamerican Journal of Psychology, 42(3), 580-588.

Friedrich, W.N., Greenberg, M.T., \& Crnic, K. A. (1983). Short-form of the questionnaire on resources and stress. American J ournal of Mental Deficiency, 88, 41-48.

Gallimore, R., Weisner, T. S., Kaufman, S. Z., \& Bernheimer, L. P. (1989). The social construction of ecocultural niches: family accommodation of developmentally delayed children. American J ournal on Mental Retardation, 94(3), 216-230.

Glenn, S., Cunningham, C., Poole, H., Reeves, D., \& Weindling, M. (2008). Maternal parenting stress and its correlates in families with a young child with cerebral palsy. Child: Care, Health and Development, 35(1), 71-78.

Haase, V. G., Barreto, G. V., \& Freitas, P. M. (2009). Adaptação psicossocial de famílias de crianças com transtornos do desenvolvimento. In: V.G. Haase \& J. F. Penna(Org.). Aspectos Biopsicossociais da Saúde na Infância e Adolescencia. Belo Horizonte: COOPMED.

Hastings, R. P. (2002) Parental stress and behaviour problems of children with developmental disability. Journal of Intellectual and Developmental Disability, 27, 149-160.

Hartley, H., \& Thomas, J. E. (2003). Current practice in the management of children with cerebral palsy: a national Survey of pediatric dietitians. Journal Human Nutrition Dietetics, 16(4), 219-224.

Hauser-Cram, P., Warfield, M. E., Shonkoff, J. P., Krauss, M. W., Sayer, A., Upshur, C. C., \& Hodapp, R. M. (2001). Children with disabilities: A longitudinal study of child development and parent well-being. Monographs of the Society for Research in Child Development, i-126. 
Ketelaar, M., Volman, M. J. M., Gorter, J. W., \& Vermeer, A. (2008) Stress in parents of children with cerebral palsy: what sources of stress are we talking about? Child: Care, Health and Development, 34(6), 825-829.

King, S., Teplicky, R., King, G., \& Rosenbaum P. (2004). FamilyCentered Service for Children With Cerebral Palsy and Their Families: A Review of the Literature. Seminars in Pediatric Neurology, 11(1), 78-86.

Lach, L. M., Kohn, D. E., Gardner, R. E., Brehaut, J. C., Miller, A. R., Klassen, A. F., \& Rosenbaum, P. L. (2009). The health and psychosocial functioning of caregivers of children with neurodevelopmental disorders. Disability and Rehabilitation, 31(8), 607-618.

Lai, M. C., \& Yang, S. N. (2010). Perinatal Hypoxic-Ischemic Encephalopathy. Journal of Biomedicine and Biotechnology, 2011, 1-6.

Lazarus, R. S., \& Folkman, S. (1984). Stress, Appraisal and Coping. New York: Springer.

Lin, J. P. (2003). The cerebral palsies: a physiological approach. J ournal of Neurology, Neurosurgery \& Psychiatry, 74(1), 9-23.

Manuel, J., Naughton, M. J., Balkrishnan, R., Smith, B. P., \& Koman, L. A. (2003). Stress and adaptation in mothers of children with cerebral palsy. J ournal of Pediatric Psychology, 28(3), 197-201.

Miner, J. L., \& Clarke-Stewart, K. A. (2008) Trajectories of externalizing behaviour from age two to age nine: relations with gender, temperament, ethnicity, parenting and rater. Developmental Psychology, 44(3), 771-786.

Miodrag, N., \& Hodapp, R.M. (2010). Chronic stress and health among parents of children with intellectual and developmental disabilities. Current Opinion in Psychiatry, 23, 407-411.

Mobarak, R., Khan, N. Z., Munir, S., Zaman, S. S., \& McConachie, H. (2000). Predictors of stress in mothers of children with cerebral palsy in Bangladesh. Journal of Pediatric Psychology, 25(6), 427-433.

Monteiro, C. B. M, Jakabi, C.M., Palma, G.C.S., Pasin, C.T., \& Meira C.M.J. (2010). Aprendizagem motora em crianças com paralisia cerebral. Revista Brasileira de Ciência e Movimento, 20(2), 250262.

Murphy, N. A., Christian, B., Caplin, D. A., \& Young, P. C. (2007). The health of caregivers for children with disabilities: Caregiver perspectives. Child: Care, Health and Development, 33(2), 180- 187.

Mutch, L., Alberman, E., Hagberg, B., Kodama, K., \& Perat, M. V. (1992). Cerebral palsy: palsy epidemiology: Where have we been and where going? Developmental Medicine \& Child Neurology, 34(6), 547-551. 
Nereo, N. E., Fee, R. J., \& Hinton, V. J. (2003). Parental stress in mothers of boys with Duchenne muscular dystrophy. Journal of Pediatric Psychology, 28(7), 473-484.

Parkes, J., Whote-Koning, M., Dickinson, H. O., Thyen, U., Arnaud, C., Beckung, E., Fauconnier, J., Marcelli, M., Mcmanus, V., Michelsen, S. I., Parkinson, K., \& Colver, A. (2008). Psychological problems in children with cerebral palsy: a cross sectional European study. Journal of Child Psychology and Child Psychiatry, 49, 405-413.

Parkes, J., Caravale, B., Marcelli, M., Franco, F., \& Colver, A. (2011). Parenting stress and children with cerebral palsy: a European cross-sectional survey. Developmental Medicine \& Child Neurology, 53, 815-821.

Pasquali, L., Gouveia, V. V., Andriola, W. B., \& Ramos, A. L. (1996). Questionário de Saúde Geral de Goldberg. Manual Técnico. São Paulo, SP: Casa do psicólogo, 1996.

Pedersen, S. D., Parson, H. G., \& Dewey, D. (2004). Stress levels experienced by the parents of enterally fed children. Child: Care, Health and Development, 30(5), 507-513.

Pinheiro, M. I. S., Haase, V. G., Amarante, C. L. D., Prette, A. D., \& Del Prette, Z. A. P. D.(2006). Treinamento de habilidades sociais e educativas para pais de crianças com problemas de comportamento. Psicologia: Reflexão e Crítica, 19, 407-414.

Pitman, E., \& Matthey, S. (2004). The SMILES program: A group program for children with mentally ill parents or siblings. American J ournal of Orthopsychiatry, 74(3), 383-388.

Phelps, K. W., Mccammon, S. L., Wuensch, K. L., \& Golden, J. A. (2009). Enrichment, stress, and growth from parenting an individual with an autismo spectrum disorder. Journal of Intellectual \& Developmental Disability, 34(2), 133-141.

Ribeiro, M. F. M., Porto, C. C., \& Vandenberghe, L. (2013). Parental stress in families of children with cerebral palsy: an integrative review. Ciência \& Saúde Coletiva, 18(6), 1705-1715.

Raina, P., O'Donnell, M., Rosenbaum, P., Brehaut, J., Water, S.D., Russell, D., Swinton, M., Zhu, B., \& Wood, E. (2005). The helth and well-being of caregivers of children with cerebral palsy. Pediatrics, 115(6), e626-e636.

Schuengel, C., Rentinck, I. C. M., Stolk, J., Voorman, J. M., Loots, G. M. P., Ketelaar, M., Gorter, J. W., \& Becher, J. G. (2009). Parents' reactions to the diagnosis of cerebral palsy: associations between resolution, age and severity of disability. Child: Care, Health and Development, 35(5), 673-680.

Sigurdardottir, S., Indredavik, M. S., Eiriksdottir, A., Einarsdottir, K., Gudmundsson, H. S., \& Vik, T. (2010). Behavioural and emotional symptoms of preschool children with cerebral palsy: 
a population-based study. Developmental Medicine \& Child Neurology, 52(11), 1056-1061.

Sipal, R. F., Schuengel C., Voorman J.M., Van Eck M., \& Becher, J.G. (2010). Course of behaviour problems of children with cerebral palsy: the role of parental stress and support. Child: Care, Health and Development, 36 (Suppl. 1), 74-84.

Skok, A., Harvey, D., \& Reddihough, D. (2006). Perceived stress, perceived social support, and wellbeing among others of schoolaged children with cerebral palsy. Journal of Intellectual \& Developmental Disability, 31(1), 53-57.

Sofronoff, K., \& Farbotko, M. (2002). The Effectiveness of Parent Management Training to Increase Self-Efficacy in Parents of Children with Asperger Syndrome. Autism, 6(3), 271-286.

Taanila, A., Syrjälä, L., Kokkonen, J., \& Jarvelin, L. M. (2002). Coping of parentes with physically and/or intellectually disabled children. Child: Care, Helth e Development, 28(1), 73-86.

Thompson, R. J., Gil, K. M., Gustafson, K. E., George, L. K., Keith, B. R., Spock, A., \& Kinney, T. R. (1994). Stability and change in the psychological adjustment of mothers of children and adolescents with cystic fibrosis and sickle cell disease. Journal of Pediatric Psychology, 19(2), 171-188.

Yim, S. Y., Moon, H. W., Rah, U. W., \& Lee, I. I. Y. (1996). Psychological characteristics of mothers of children with desabilities. Yonsei Medical Journal, 37(6), 380-384.

\section{Endereço para correspondência \\ Patrícia Martins de Freitas}

Instituto Multidisciplinar em Saúde da Universidade Federal da Bahia

Rua Rio de Contas, 58 Quadra 17 Lote 58, Candeias, CEP 45029-094, Vitória da Conquista - BA, Brasil

Endereço eletrônico: pmfrei@gmail.com

\section{Carine Mendes Rocha}

Centro de Ciências da Saúde da Universidade Federal do Recôncavo da Bahia Avenida Carlos Amaral, 1015, Cajueiro, CEP 44570-000, Santo Antônio de Jesus BA, Brasil

Endereço eletrônico: carimrocha@yahoo.com.br

\section{Vitor Geraldi Haase}

Departamento de Psicologia da Universidade Federal de Minas Gerais

Avenida Antônio Carlos, 6.627, Campus Pampulha, CEP 31270-901, Belo Horizonte

- MG, Brasil

Endereço eletrônico: vghaase@gmail.com

Recebido em: 14/02/2012

Reformulado em: 02/04/2014

Aceito para publicação em: 04/04/2014

\section{Notas}

* Professora Adjunta do Instituto Multidisciplinar em Saúde da Universidade Federal da Bahia. Doutora em Ciências da Saúde: Saúde da Criança e do Adolescente, 
Patrícia Martins de Freitas, Carine Mendes Rocha, Vitor Geraldi Haase Análise dos preditores do estado psicológico das mães de crianças com Paralisia

Cerebral

Coordenadora do Núcleo de Investigações Neuropsicológicas da Infância e Adolescência do IMS-UFBA.

** Psicóloga da Universidade Federal do Recôncavo da Bahia. Graduada em Psicologia pela Universidade Federal do Recôncavo da Bahia.

*** Professor adjunto do Departamento de Psicologia da Universidade Federal de Minas Gerais. Doutor em Psicologia Médica. Mestre em Lingüística Aplicada. Graduado em Medicina. Programa de Pós-graduação em Saúde da Criança e do Adolescente da Faculdade de Medicina da UFMG, Programa de Pós-graduação em Neurociências do Instituto de Ciências Biológicas da UFMG. 